\title{
Observation of ion Coulomb crystals in a cryogenic linear octupole rf ion trap
}

\author{
K. Okada, ${ }^{1, *}$ T. Takayanagi, ${ }^{1}$ M. Wada, ${ }^{2}$ S. Ohtani, ${ }^{3}$ and H. A. Schuessler ${ }^{4}$ \\ ${ }^{1}$ Department of Physics, Sophia University, 7-1 Kioicho, Chiyoda, Tokyo 102-8554, Japan \\ ${ }^{2}$ Atomic Physics Laboratory, RIKEN, 2-1 Hirosawa, Wako, Saitama 351-0198, Japan \\ ${ }^{3}$ Institute for Laser Science (ILS), University of Electro-Communications, 1-5-1 Chofugaoka, Chofu, Tokyo 182-8585, Japan \\ ${ }^{4}$ Department of Physics, Texas A\&M University, College Station, Texas 77843, USA \\ (Received 21 April 2009; revised manuscript received 6 July 2009; published 7 October 2009)
}

\begin{abstract}
Ion Coulomb crystals of laser-cooled $\mathrm{Ca}^{+}$ions have been observed in a cryogenic linear octupole rf ion trap for the first time. Due to the almost field-free central region, large ion crystals containing over $10^{4}$ ions of millimeter size were successfully produced. The shapes of the crystals can be manipulated by changing the static voltages applied to the octupole rods and the endcap electrodes. Quasi-two-dimensional Coulomb crystals consisting of a small number of ions were also observed with single-ion sensitivity. When a large number of ions is crystallized in the octupole trap, a new kind crystal image with a dip along the axial direction was obtained. The results show that a linear octupole trap and multipole traps are available for producing large, cold, and novel ion Coulomb crystals. The planar ring-type crystal which has not been observed before may lead to future interesting applications.
\end{abstract}

DOI: 10.1103/PhysRevA.80.043405

PACS number(s): $37.10 . T y, 37.10 . R s, 42.50 . D v, 52.27 . J t$

Much effort is currently directed toward the realization of scalable quantum systems. Ion Coulomb crystals of lasercooled ions are promising tools for such larger scale quantum information processing and quantum computing $[1,2]$. In addition high-density and large ion crystals are ideal for studying ultracold ion-molecule collisions [3]. The interest extends also to cavity QED [4], to tests of a possible variation in the fundamental physical constants [5], and to precision laser spectroscopy of unstable nuclear ions [6]. So far systematic studies of ion Coulomb crystals have been performed in Penning traps and Paul rf ion traps including their linear configurations [7-10]. Recently we have shown that a linear multipole rf trap can be used to also produce ion Coulomb crystals [11]. In essence, a large number of cold ions can be trapped in the larger trapping volume than that of a linear quadrupole trap of comparable size. Actually, the large almost field-free region in the middle of the multipole traps is of advantage for studies of low temperature ion-molecule reactions [12], high-resolution rotational spectroscopy [13], and highly stable ion clocks [14] using buffer-gas-cooled ions. In spite of this, sufficient attention to ion Coulomb crystals in a linear if multipole ion trap has not yet been paid except in our previous work [11]. The ion Coulomb crystals in a linear multipole trap have interesting features as well as the buffer-gas-cooled ions, and some of those do not occur in a linear quadrupole rf ion trap.

For instance, large numbers of ions of over $10^{4}$ can be readily cooled to less than $10 \mathrm{mK}$ and large Coulomb crystals with millimeter size are easily produced as described later. These properties are suitable for measuring cold ionmolecule reaction rates. The study of cold ion-polar molecule collisions is also a project to apply such cold large ion crystals [3]. We have the plan to study cold ion-polar molecule reactions, such as $\mathrm{Ca}^{+}+\mathrm{NH}_{3}\left(\mathrm{ND}_{3}\right)$, by combining a Stark decelerator [15] with a cryogenic multipole trap. In

\footnotetext{
*okada-k@sophia.ac.jp
}

such experiments sympathetically cooled molecular ions can also be used as a cold ion target.

Moreover ion Coulomb crystals in a linear $2 n$-multipole trap are fascinating research objects because new features of Coulomb crystals not present in a linear quadrupole trap arise due to the $r^{2 n-2}$ dependence of the pseudopotential. The ideal sum potential of the radial pseudopotential and the static potentials for axial confinement of ions provides new kinds of crystal structures, such as ring- and cylinder-type crystals [11]. In particular the observed planar ring-type crystals have the potential to realize the proposed displacement $[16]$ and other hot gates, which operate without ground state cooling. When placed in a superimposed magnetic field for rotation, a quantum register or a quantum generator may result.

In this paper we report the first direct observation of Coulomb crystals of laser-cooled ions in a cryogenic linear octupole rf ion trap. By slightly changing the static trap voltages we have shown that the Coulomb crystals can be easily modified to exhibit various shapes. For relatively small axial static voltages $\left(V_{z}\right)$ long prolate shapes of the ion crystals are observed when alternately applying negative dc voltages $\left(V_{d c}\right)$ as well as phase-inverted rf voltages to the trap rods. On the other hand, Coulomb crystals with spherical symmetry are observed when relatively large axial voltages $V_{z}$ and positive $V_{d c}$ are applied to the electrodes. In the cylindrically symmetric case of the potential $\left(V_{d c}=0\right)$ the projections of cylinderlike crystals are observed. Moreover quasi-twodimensional Coulomb crystals consisting of a small number of ions were imaged with single-ion sensitivity.

The experimental setup is as follows. To observe Coulomb crystals of laser-cooled ions, we developed a compact octupole trap as follows. The octupole trap consists of eight rod electrodes with a diameter of $2 \mathrm{~mm}$. The distance from the trap center axis to the surface of the electrodes is $4.5 \mathrm{~mm}$, which is larger than the normal value $\left(r_{0}=3.0 \mathrm{~mm}\right)$ [12] to increase the field of view. We can adjust the position of an imaging lens system $(\times 6$ magnification $)$ outside the vacuum. The vacuum chamber enclosing the ion trap is com- 
$1.7 \mathrm{~mm}$
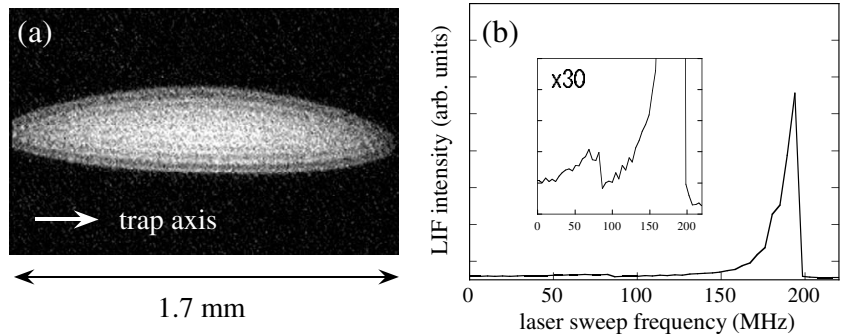

FIG. 1. Observation of (a) the crystal image and (b) the LIF spectrum of the same crystal with $\sim 1.7 \times 10^{3}$ ions. The CCD image is observed at the peak of the spectrum. The spectrum is very smooth although a very small LIF drop at about $90 \mathrm{MHz}$ is seen shown in the inset of (b). The rf frequency $\Omega=2 \pi \times 6.0 \mathrm{MHz}$ and the amplitude $V_{a c}=154 \mathrm{~V}$ were applied. The static voltages are $V_{d c}=-1.5 \mathrm{~V}, V_{z 1}=0.95 \mathrm{~V}$, and $V_{z 2}=0.50 \mathrm{~V}$, respectively. The $\mathrm{CCD}$ exposure time is $3 \mathrm{~s}$.

posed of two vacuum pumps (ion pump and turbo molecular pump) and a compact miniature UHV chamber (3.2 in. diameter and $1.38 \mathrm{in}$. height). In addition, the ion trap is mounted on a vessel containing liquid nitrogen to obtain an ultrahigh vacuum lower than $10^{-8} \mathrm{~Pa}$. This cryogenic vessel is indispensable for stable observation of ion Coulomb crystals.

Rf voltages of inverted phases are alternately applied to adjacent rod electrodes of the octupole trap. A typical frequency and amplitude are $f=6.0 \mathrm{MHz}$ and $V_{a c}=160 \mathrm{~V}$, respectively. It is pointed out that this larger than ideal [12] trap configuration produces with larger rf voltages (1.5 times larger amplitude) an almost ideal effective potential. Static axial voltages $\left(V_{z 1}, V_{z 2}\right)$ of up to a few volts for the axial confinement are normally applied to the cylindrical endcap electrodes with an inner diameter of $6 \mathrm{~mm}$. The distance between the endcaps is $36 \mathrm{~mm}$ and the total length of the trap is $54 \mathrm{~mm}$. We also alternately applied a positive and negative static bias voltage $V_{d c}$ to the octupole rods when necessary.

For laser cooling of the $\mathrm{Ca}^{+}$ions two grating stabilized laser diodes $(\lambda=397$ and $866 \mathrm{~nm})$ are used. The details are described in Ref. [11]. The laser-induced fluorescence (LIF) at $397 \mathrm{~nm}$ is observed by a cooled charge coupled device (CCD) camera at a right angle to the trap axis. The camera with the lens system is mounted on a precision stage to adjust the image position of a Coulomb crystal. An UV filter is mounted to the entrance of the lens system to reduce background photons from the room light. A typical CCD exposure time is $1-10 \mathrm{~s}$, which is long enough to average out the micromotion effect of the Coulomb crystal images.

In Fig. 1 the result of observations of the LIF spectrum and the crystal image on the same crystal is shown. The CCD image is observed near the peak in the LIF spectrum of Fig. 1(b). The linewidth of the spectrum is comparable to the half of the natural linewidth of $22 \mathrm{MHz}$ in the ${ }^{2} S_{1 / 2^{-}}{ }^{2} P_{1 / 2}$ transition [17]. Thus the corresponding ion temperature is estimated to be lower than $9 \mathrm{mK}$. This is a conservative estimate since the ion temperature is at a minimum around the peak position. A pronounced fluorescence dip, which is normally in a quadrupole trap associated with the phase transition from the cloud state to the liquid and crystal states, was not
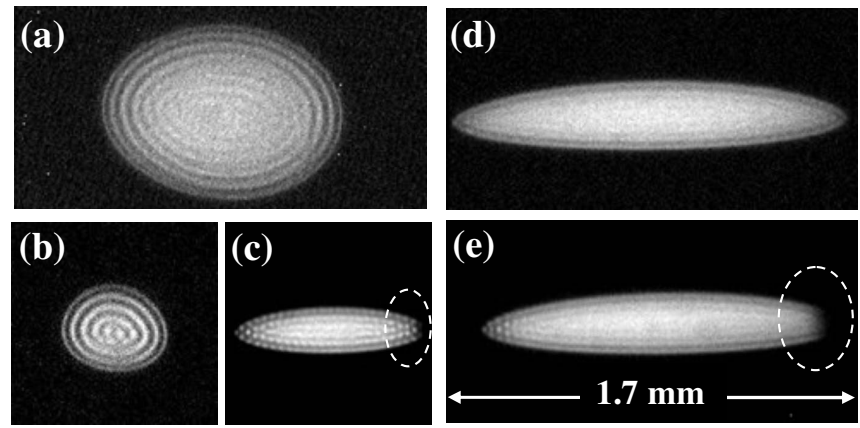

FIG. 2. Pictures of Coulomb crystals under different trapping conditions. The trap $z$ axis is along the horizontal direction of the images. The scale of all images is same. The lasers were irradiated from the right of the image. The rf voltage and the static voltages are (a) $f=6.04 \mathrm{MHz}, V_{a c}=157 \mathrm{~V}, V_{z 1}=V_{z 2}=0.10 \mathrm{~V}, V_{d c}=0 \mathrm{~V}$, (b) $f=6.04 \mathrm{MHz}, V_{a c}=157 \mathrm{~V}, V_{z 1}=V_{z 2}=3.40 \mathrm{~V}, V_{d c}=+0.70 \mathrm{~V}$, (c) $f=6.0 \mathrm{MHz}, V_{a c}=164.5 \mathrm{~V}, V_{z 1}=V_{z 2}=2.60 \mathrm{~V}, V_{d c}=-2.51 \mathrm{~V}$, (d) $f=5.97 \mathrm{MHz}, V_{a c}=169.0 \mathrm{~V}, V_{z 1}=V_{z 2}=0.60 \mathrm{~V}, V_{d c}=-2.52 \mathrm{~V}$, (e) $f=6.0 \mathrm{MHz}, V_{a c}=164.5 \mathrm{~V}, V_{z 1}=V_{z 2}=1.00 \mathrm{~V}, V_{d c}=-2.37 \mathrm{~V}$. The numbers of ions are estimated to be (a) $4 \times 10^{3}$, (b) $4 \times 10^{2}$, (c) $2 \times 10^{2}$, (d) $9 \times 10^{2}$, and (e) $7 \times 10^{2}$, respectively. The dotted line circles in (c) and (e) show the dark region of the Coulomb crystals due to sympathetically cooled ions $\left({ }^{44} \mathrm{Ca}^{+}\right)$.

observed for the octupole trap. Actually a very small LIF drop at about $90 \mathrm{MHz}$ is seen as shown in the inset of Fig. 1(b). The smooth spectrum suggests that the rf heating effect is rather small in the rf octupole trap since it is considered to occur as a result of a competition between the rf heating and the laser-cooling effect [10]. In the present octupole trap we observe pronounced fluorescence dips only when a relatively large number of ions $\left(>10^{4}\right)$ are trapped and laser cooled (see Fig. 5).

As shown in Fig. 1(a) a prolate crystal with shell structure was observed. The length of the crystal is more than $1.7 \mathrm{~mm}$. Assuming cylindrical symmetry and using a uniform number density $\left(1.3 \times 10^{7} \mathrm{~cm}^{-3}\right)$ derived from the small Coulomb crystal shown in Fig. 4, the number of ions is estimated to be $\sim 1.7 \times 10^{3}$.

Coulomb crystals observed under different conditions are depicted in Fig. 2. One recognizes that the crystal structure can be easily modified by changing the static voltages of $V_{z}$ and $V_{d c}$. Long prolate shapes of the ion crystals are observed when applying a large negative $V_{d c}$ [Figs. 2(c)-2(e)], while spherical shapes are observed, when a small positive $V_{d c}$ and a relatively large $V_{z}$ are applied [Figs. 2(a) and 2(b)]. Although the trapping pseudopotential is generated by the octupole rf fields, the crystals are similar to the ones observed in linear quadrupole traps. The reason is that the alternately applied $V_{d c}$ voltages generate a local potential minimum of quadratic form $\left(\propto r^{2}\right)$ along the radial direction to the rods to which $V_{d c}$ is applied since the flat pseudopotential $\left(\propto r^{6}\right)$ is modified by the additional dc voltages [12]. That is, the storage volume of ions is reduced in the radial direction as compared to the case of $V_{d c}=0$. Thus, an ion crystal is formed with a long prolate shape by applying negative $V_{d c}$. Figure 3 displays relevant potential curves, where (a) potential versus $r$ for selected radial directions (the inset defines the angle $\phi$ ) 

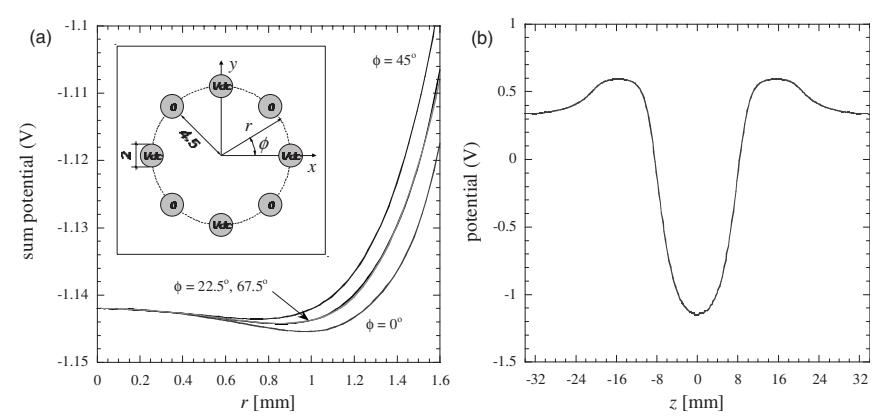

FIG. 3. Sum potential of the pseudopotential of the octupole trap and the calculated realistic potential at $V_{d c}=-2.6 \mathrm{~V}$ and $V_{z}$ $=0.6 \mathrm{~V}$ [similar condition of Fig. 2(d)]. The latter potential is numerically calculated using SIMION3D code [18]. (a) Radial potential curves for the selected directions $\phi$, which are defined in the inset. (b) Calculated potential along $z$ direction at the minimum of $\phi$ $=0^{\circ}$ when applying only $V_{z}=0.6 \mathrm{~V}$.

and (b) potential versus $z$ at the minimum of $\phi=0^{\circ}$ in Fig. 3(a) along the trap center. This modified potential and the radiation pressure force by the lasers could be the reason why asymmetry of the crystal image of Fig. 2(b) is seen. It is to be noted that a crystal image slightly moves up and down by changing $V_{d c}$ since the potential minimum in the radial direction shifted (Fig. 3). Thus the images in Fig. 2 were obtained by adjusting the position of the CCD camera along the vertical axis.

The shell structure is clearly observed in Fig. 2(a) even though the radial size of the crystal is larger than one millimeter. The intervals between the shells range from 40 to $50 \mu \mathrm{m}$. This crystal image demonstrates that the ions are cooled to very low temperatures and that the rf heating effect is very small in a large part of the radial trapping region. In addition dark regions on the CCD images caused by impurity ions are observed on the right edges of Figs. 2(c) and 2(e), while the similar crystal without impurities shown in Fig. 2(d) has a symmetric shape. The impurities are mainly ${ }^{44} \mathrm{Ca}^{+}$ ions since a $\mathrm{Ca}$ piece containing natural isotopes is used in the present experiment. With the cooling lasers incident from the right the radiation pressure pushes at resonance the ${ }^{40} \mathrm{Ca}^{+}$ crystal to the left side of the image. Due to the fact that the impurity ions do not experience radiation forces, they accumulate in the right, while simultaneously they are sympathetically cooled. These results indicate that sympathetic cooling can also be executed to a linear octupole rf ion trap.

Small planar Coulomb crystals were also observed with single-ion sensitivity and are depicted in Fig. 4. Surprisingly, the ions do not form a Coulomb chain, which is the typical form observed in a linear quadrupole trap, but form an oval shape with the ion-ion distance of about $70 \mu \mathrm{m}$ without $V_{d c}$ voltage. The images indicate that quasi-two-dimensional Coulomb crystals were produced in the $z-r$ plane in the octupole trap. These arrangements, however, do not correspond to the simulation results of Ref. [11], in which planar ring Coulomb crystals are produced for small numbers of ions in the $r$ plane. A linear crystal image along the $r$ direction is expected by the simulation. The reason for the deformed shapes can be attributed to the slight misalignment of the octupole rods and the patch effect of electric charges. Our
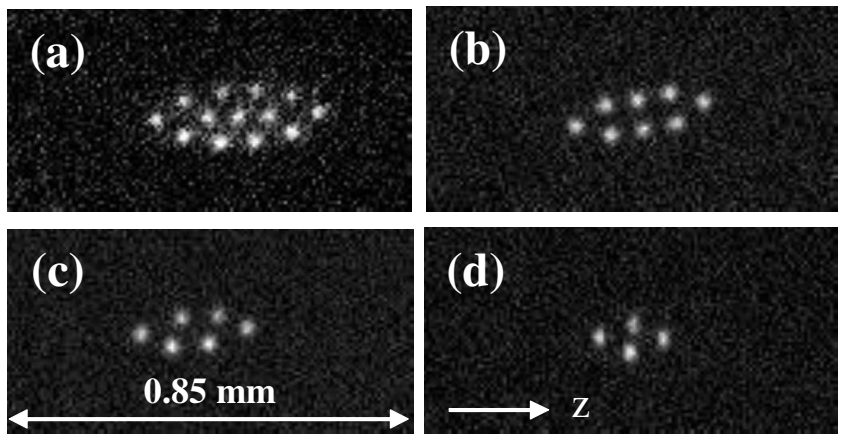

FIG. 4. Planar images of $\mathrm{Ca}^{+}$Coulomb crystals consisting of a small number of ions. The rf voltage and the static voltage are $f$ $=6.04 \mathrm{MHz}, V_{a c}=157 \mathrm{~V}$, and $V_{d c}=0 \mathrm{~V}$, respectively. The scale of all images is same. (a) $N_{i o n}=13, V_{z 1}=V_{z 2}=0.50 \mathrm{~V}$, (b) $N_{\text {ion }}=8$, $V_{z 1}=V_{z 2}=0.10 \mathrm{~V}$, (c) $N_{i o n}=6, V_{z 1}=V_{z 2}=0.30 \mathrm{~V}$, (d) $N_{\text {ion }}=4, V_{z 1}$ $=V_{z 2}=0.85 \mathrm{~V}$.

experimental results suggest that careful arrangement of electrodes and precise control of the static voltages $\left(V_{d c}, V_{z}\right)$ is necessary in order to keep the cylindrical symmetry of the flat multipole pseudopotentials and to produce few ion ring Coulomb crystals in the $r$ plane. A smaller octupole trap and a hexapole arrangement will help us to maintain the symme-
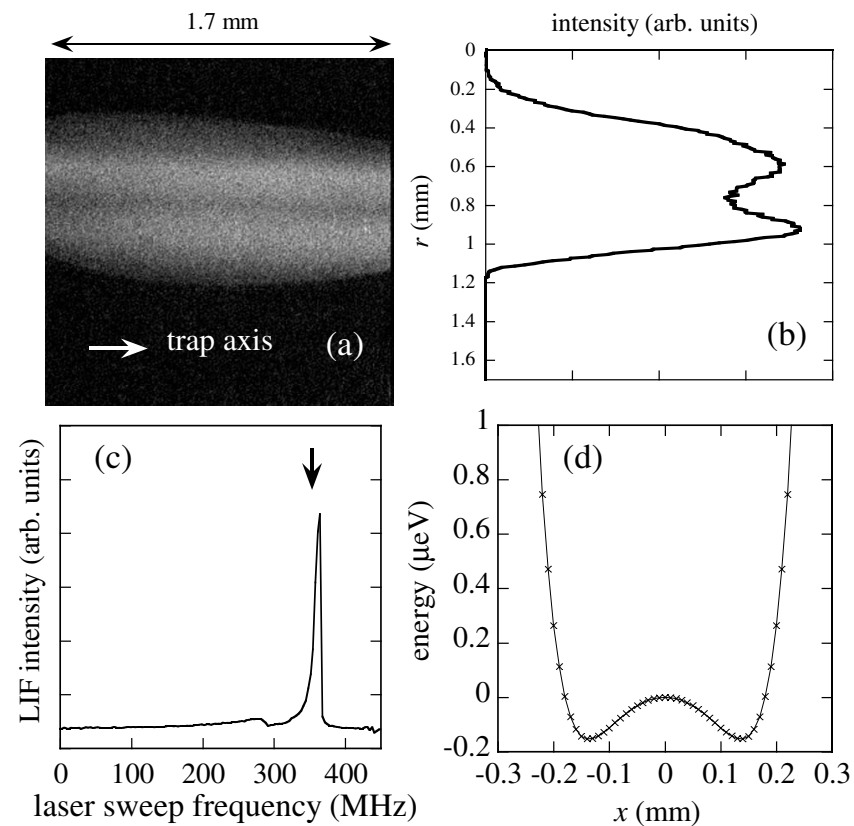

FIG. 5. (a) CCD image of a large Coulomb crystal containing more than $10^{4}$ ions. The $\mathrm{rf}$ voltage and the static voltages are $f$ $=6.04 \mathrm{MHz}, V_{a c}=157 \mathrm{~V}, V_{d c}=0 \mathrm{~V}$, and $V_{z}=0.8 \mathrm{~V}$, respectively. (b) Profile of the projection of the image shown in (a) on the vertical axis. (c) Laser cooling spectrum measured at the same time as the CCD image (a). During the laser sweep in (c) the ion crystal should change from a cloud to a crystal to a cloud. The ion temperature in the crystal state is estimated to be less than $9 \mathrm{mK}$ since the spectral width is comparable to the half of the natural width of the ${ }^{2} S_{1 / 2^{-}}{ }^{2} P_{1 / 2}$ transition. (d) Calculated sum potential of the pseudopotential of the octupole trap and the axial static potential. The same trapping parameters as the experiment was used for the calculation. The geometric factor of the axial static potential was assumed to be $\kappa=0.01$ [11]. 
try and reduce the perturbations since in such a lower-order multipole trap stronger rf fields are present.

When a much larger number of ions is crystallized, a new kind of crystal image was observed as shown in Fig. 5(a). The number of ions is estimated to be more than $10^{4}$. The image was observed by setting the laser frequency to the position indicated by the arrow in the laser cooling spectrum of Fig. 5(c). Although the image does not resolve single ions, a small dip along the trap axial direction is recognized. In the projection profile [Fig. 5(b)] the two peaks of the fluorescence are clearly observed. The reason of this interesting property is attributed to the characteristic sum potential in the radial direction [11]. The distance between the two peaks is about $300 \mu \mathrm{m}$, which corresponds to the distance between the two minima of the sum potential shown in Fig. 5(d).

In conclusion, we have successfully observed Coulomb crystals of laser-cooled $\mathrm{Ca}^{+}$ions in a cryogenic linear octupole rf ion trap and also experimentally demonstrated that in principle a cylinderlike crystal and a ring crystal of laser- cooled $\mathrm{Ca}^{+}$ions can be produced in such a multipole trap. Because of the small micromotion amplitudes in the large almost-field-free central region of the octupole ion trap, large sizes of Coulomb crystals including more than $10^{4}$ ions can be readily produced. The shape of the crystals can also be manipulated by changing rf and static trapping voltages. The ion temperatures are estimated to be less than $10 \mathrm{mK}$ from the LIF spectra even though the number of ions is larger than $10^{4}$. Linear octupole traps and multipole traps are available for producing large, cold, and novel-type ion Coulomb crystals. The crystal forms fill a gap in present research and have a variety of possible applications [1,3-5].

This work was financially supported in part by Grant-inAid for Young Scientists (A) and (B) from the Ministry of Education, Culture, Sports, Science and Technology (MEXT) by the Matsuo Foundation and by the Robert A. Welch Foundation under Grant No. A1546.
[1] J. I. Cirac and P. Zoller, Phys. Rev. Lett. 74, 4091 (1995).

[2] C. Monroe, D. M. Meekhof, B. E. King, W. M. Itano, and D. J. Wineland, Phys. Rev. Lett. 75, 4714 (1995).

[3] S. Willitsch, M. T. Bell, A. D. Gingell, S. R. Procter, and T. P. Softley, Phys. Rev. Lett. 100, 043203 (2008).

[4] P. Herskind, A. Dantan, M. Langkilde-Lauesen, A. Mortensen, J. L. Sorensen, and M. Drewsen, Appl. Phys. B: Lasers Opt. 93, 373 (2008).

[5] U. Fröhlich, B. Roth, P. Antonini, C. Lämmerzahl, A. Wicht, and S. Schiller, Lect. Notes Phys. 648, 297 (2004).

[6] K. Okada, M. Wada, T. Nakamura, A. Takamine, V. Lioubimov, P. Schury, Y. Ishida, T. Sonoda, M. Ogawa, Y. Yamazaki, Y. Kanai, T. M. Kojima, A. Yoshida, T. Kubo, I. Katayama, S. Ohtani, H. Wollnik, and H. A. Schuessler, Phys. Rev. Lett. 101, 212502 (2008).

[7] S. L. Gilbert, J. J. Bollinger, and D. J. Wineland, Phys. Rev. Lett. 60, 2022 (1988).

[8] R. Blümel, C. Kappler, W. Quint, and H. Walther, Phys. Rev. A 40, 808 (1989).
[9] D. H. E. Dubin and T. M. O’Neil, Rev. Mod. Phys. 71, 87 (1999).

[10] L. Hornekaer and M. Drewsen, Phys. Rev. A 66, 013412 (2002).

[11] K. Okada, K. Yasuda, T. Takayanagi, M. Wada, H. A. Schuessler, and S. Ohtani, Phys. Rev. A 75, 033409 (2007).

[12] D. Gerlich, Adv. Chem. Phys. 82, 1 (1992).

[13] O. Asvany, O. Ricken, H. S. P. Muller, M. C. Wiedner, T. F. Giesen, and S. Schlemmer, Phys. Rev. Lett. 100, 233004 (2008).

[14] L. Maleki and J. D. Prestage, Lect. Notes Phys. 648, 331 (2004).

[15] S. A. Rangwala, T. Junglen, T. Rieger, P. W. H. Pinkse, and G. Rempe, Phys. Rev. A 67, 043406 (2003).

[16] J. I. Cirac and P. Zoller, Nature (London) 404, 579 (2000).

[17] NIST Atomic Spectra DataBase Ver, 3.1.0, http:// physics.nist.gov/PhysRefData/ASD/index.html

[18] SIMION 3D Version 8.0, Scientific Instrument Services Inc., Idaho National Laboratory. 